\title{
Comparison of postoperative changes in radial peripapillary capillary density between femtosecond laser-assisted cataract surgery and conventional phacoemulsification surgery using optical coherence tomography angiography
}

Hong Zhang ( $\nabla$ tmuechong@sina.com )

Tianjin Medical University Eye Hospital

\section{Liangzhang Tan}

Tianjin Medical University Eye Hospital

\section{Fang Tian}

Tianjin Medical University Eye Hospital

\section{Xue Gong}

Tianjin Medical University Eye Hospital

\section{Lu Chen}

Tianjin Medical University Eye Hospital

\section{Zhiqiong Ma}

Zhejiang Xiaoshan Hospital

Jingli Liang

Tianjin Medical University Eye Hospital

\section{Chen Guo}

Tianjin Medical University Eye Hospital

\section{Xiaorong Li}

Tianjin Medical University Eye Hospital

\section{Research article}

Keywords: femtosecond laser-assisted cataract surgery; conventional phacoemulsification surgery; radial peripapillary capillary density; non-invasive flow density; optical coherence tomography angiography

Posted Date: May 1st, 2020

DOI: https://doi.org/10.21203/rs.3.rs-24058/v1 
License: (c) (i) This work is licensed under a Creative Commons Attribution 4.0 International License. Read Full License 


\section{Abstract}

Purpose To assess differences in post-surgical radial peripapillary capillary (RPC) density changes between femtosecond laser-assisted cataract surgery (FLACS) and conventional phacoemulsification surgery (CPS) using optical coherence tomography angiography (OCTA).

Methods. Fifty-two eyes of 52 patients were enrolled in this study. Depending on their preference, patients with age-related cataract underwent FLACS or CPS. Automated peripapillary maps and capillary density calculations were retrospectively evaluated using OCTA before surgery and on day 1 and months 1 and 3 postoperatively.

Results. The whole-image and peripapillary RPC densities were lower at day 1 and months 1 and 3 postoperatively than before surgery in the FLACS group (all $P<0.05$ ). However, there was no change in the whole-image and peripapillary RPC densities before and after surgery, across different time-points, in the CPS group (all $P>0.05$ ). Moreover, comparison of data corresponding to each time-point between the FLACS and CPS groups showed no significant differences in any metrics prior to surgery (all $P>0.05$ ). However, the whole-image and peripapillary RPC densities were significantly different at day 1 and month 1 after the surgery (all $P<0.05$ ). At the 3-month follow-up, we did not observe significant differences (all $P>0.05)$.

Conclusions. Postoperative whole-image and peripapillary RPC densities were lower in the FLACS group than in the CPS across time-points. While the short-term RPC density changes were resolved, to elucidate the long-term implications of FLACS, especially for eyes with glaucoma, diabetic retinopathy, etc., further investigations are required.

\section{Introduction}

Elevated intraocular pressure (IOP) is the leading cause of glaucomatous optic nerve impairment [1], which is characterised by a decrease in the number of retinal ganglion cells. In association with IOP elevation, mechanical compression is thought to force fluid through the laminar pores and cause the blockade of axonal flow, thereby inducing axonal injury [2]. Moreover, increased IOP can deform the capillary containing laminar beams, thereby reducing the blood supply to the laminar segments of the axons [3]. A number of studies have shown that reduced optic nerve head perfusion plays a crucial role in the pathogenesis of glaucoma $[4,5]$

In vivo and ex vivo preparations of healthy eyes suggest that IOP negatively impacts the posterior segment structures during ophthalmic procedures $[6,7]$. In femtosecond laser-assisted cataract surgery (FLACS), during the femtosecond laser pre-treatment, suction is applied to stabilise the eye of the patient on the laser platform prior to capsulorhexis, lens fragmentation and liquefaction, and corneal incision [8]. Studies on laser in situ keratomileusis (LASIK) and the femtosecond pre-treatment in cataract surgery reveal that the application of docking may cause a transient and irregular elevation of IOP [9-11]. A broad fluctuation in IOP can lead to retinal complications, including choroidal neovascularization and 
rhegmatogenous retinal detachment $[12,13]$. Especially in elderly patients with cataract, IOP increase may restrict ocular blood flow and thereby, lead to retinal ischaemic and neuropathic injury [14]. Radial peripapillary capillaries (RPCs) constitute a superficial layer of capillaries with a relatively constant calibre, and these run parallel to the retinal nerve fibre layer in the peripapillary region [15]. Considering the unique pattern and distribution of the vessels, the RPC was considered to be particularly vulnerable to elevated IOP when compared with other retinal capillaries [16]. To our knowledge, it has not been reported so far whether IOP fluctuations during FLACS can impact RPC density. Furthermore, the technical difficulties of imaging the retinal vessel density may prevent investigators from observing changes in RPC density as an early indicator of retinopathy after cataract surgery [17].

The US Food and Drug Administration-approved optical coherence tomography angiography (OCTA) is a rapidly developing technology that allows the direct and non-invasive visualisation of retinal flow density. OCTA works by tracking the motion of erythrocytes and comparing continuous B-scan signals from the same location. In addition, the technique also provides additional information on the retinal and choroidal capillary network $[18,19]$. OCTA has been previously used for the detailed quantitative and qualitative investigation of the ocular vasculature [20,21], in patients with conditions such as glaucoma, age-related macular degeneration, diabetic retinopathy, and central serous chorioretinopathy [22]. Therefore, the purpose of this study was to investigate the alternations of RPC density accompanied by fluctuations in IOP during FLACS to broaden our understanding of the safety of femtosecond pre-treatment through quantitative analyses with OCTA in elderly patients with cataracts.

\section{Materials And Methods}

\section{Study Design}

Fifty-two eyes of 52 consecutive patients with age-related cataract who underwent intraocular lens (IOL) implantation via either FLACS or CPS from August 2018 to July 2019 were included in this retrospective, interventional comparative case series. These procedures were performed uniformly at a single University Hospital (Tianjin Medical University Eye Hospital, Tianjin, China). Written informed consents were obtained from each subject based on their decision to proceed with either FLACS or CPS. The ethics committee of Tianjin Medical University approved the study, which is in accordance with the Declaration of Helsinki.

Exclusion criteria included the following: myopia or hyperopia to avoid the influence of magnification of the OCTA images, inflammatory cells in the anterior chamber $>5$ postoperation, presence of amblyopia, media opacities which prevented high-quality imaging, dense cataracts, and ocular hypertension; and a history of systemic diseases such as hypertension, diabetes mellitus and cardiovascular disease, which might interference the ocular circulation; a history of previous ocular surgery; evidence of retinal pathologies, such as retinal vascular diseases; a history of trauma; and complications such as large fluctuation of blood pressure and posterior capsular rupture during operation. 


\section{Patient metrics}

Each participant underwent a comprehensive preoperative ophthalmic examination. Assessed metrics included the best-corrected visual acuity (BCVA); the IOP, as measured by Goldmann applanation tonometry; a refractive status assessment, as determined by an automatic refractometer; slit-lamp examination; and fundoscopy. Axial length $(A L)$ was measured using the IOL Master system (Carl Zeiss, Meditec, Germany). Corneal topography was measured with a Scheimpflug device (Pentacam, Oculus Optikger ate $\mathrm{GmbH}$ ). Endothelial cell counts were determined with a specular microscope (SP-1P, Topcon Europe Medical B.V., Netherlands). B-scan ultrasound recordings were also documented (AVISO, Quantel Medical, Clermont-Ferrand, France). The macula was examined using swept-source optical coherence tomography (SS-OCT, Topcon DRI OCT-1 Atlantis). Refraction data were converted to spherical equivalents (SE; SE = spherical component $+1 / 2$ cylindrical component). Any performed procedures or analyses were conducted by an optometrist or technician.

\section{OCTA image acquisition and processing}

OCTA images were obtained using RTVue XR OCT (Optovue, Inc., Fremont, CA, USA; Software V.2017.1.0.155). The split-spectrum amplitude decorrelation algorithm (SSADA) was used to extract OCTA images, which operates A-scan of 70, $000 \mathrm{HZ}$ scans per second. A $4.5 \times 4.5 \mathrm{~mm}$ angio disc mode was centred on the optic disc, and two repeated volumes comprised by 304 B-scans were captured. During image processing, the Motion Correction Technology function was used to correct the horizontal and vertical scans for eye movement [23]. Automatic segmentation was performed by the Optovue software to generate face projection images. All scans with layer segmentation errors, signal strength index $<50$, or significant motion artefacts in images were excluded to ensure the accuracy of the measurement. Collection of data on BCVA and IOP and OCTA image acquisition and processing for this study occurred before the surgery and at day 1 and months 1 and 3 after the surgery.

The Optovue software, equipped with the density function, was used to analyse a $4.5 \times 4.5 \mathrm{~mm}$ optic disc angiogram. The average vessel density was automatically captured from the optic disc segment that extended from the internal limiting membrane (ILM) to $150 \mu \mathrm{m}$ below the ILM. The average peripapillary RPC density was automatically calculated in a 0.75-mm-wide elliptical annulus region that extended from the optic disc boundary. The peripapillary RPC density was separately processed in two regions (superiorhemi, inferior-hemi). The software further calculated the whole image vessel density. (Figure 1)

\section{Femtosecond laser-assisted pre-treatment and cataract surgical procedures}

The femtosecond laser-assisted pre-treatment for the FLACS group was performed by a surgeon, who had previously performed at least 30 FLACS procedures before operating in this study, using a femtosecond laser (Lensx, Alcon Laboratories, Inc.). Before the femtosecond laser pre-treatment trial, the same surgeon received comprehensive training. The LenSx system consists of a $50 \mathrm{kHz}$ femtosecond 
infrared laser with a pulse width of 600-800 femtoseconds, a central laser wavelength of 1,030 nm, and a maximum pulse energy of $15 \mu \mathrm{J}$. The laser pulse energy setting was $6 \mu \mathrm{J}$ for performing the anterior capsulotomy (diameter: $5.2 \mathrm{~mm}$ ). This laser is combined with a 3D spectral-domain OCT system providing visualization of the entire anterior segment during the surgical procedure and a liquid-free curved patient interface (SoftFit). The femtosecond laser system was used for capsulotomy, lens fragmentation, and corneal incision. In cases where issues with the laser treatment were noted (e.g., a repeated inability to dock, laser machine fault, etc.) and when it could not be operated, patients instead underwent CPS. All cataract surgical procedures were performed under local anaesthesia.

After the femtosecond laser treatment, each participant was transferred to another operating room for the remainder of the procedure. Phacoemulsification was completed using torsional phacoemulsification on an active-fluidics-based platform (Centurion ${ }^{\circledR}$ Alcon Laboratories, Inc., USA). Patients undergoing CPS were prepared for surgery in the same way as those in the femtosecond laser arm. Instead of receiving laser pre-treatment, they directly underwent the operation. The default IOL used in the capsular bag was a hydrophobic acrylic IOL (Alcon Laboratories, Inc., USA). Patients received levofloxacin (Cravit) and prednisolone acetate 1\% (Pred Forte) eyedrops four times a day for 1-week after the surgery, followed by tapering for 3 weeks for both groups.

\section{Statistical Analysis}

The normality of data distribution was tested using the Kolmogorov-Smirnov test. After confirmation of the normality assumption, data are generally presented as mean \pm standard deviation (SD) values. Within-subject comparisons of RPC density, BCVA, and IOP at follow-up and baseline were performed using the paired samples t-test. Between-group time-course differences of RPC density, BCVA, and IOP between the two treatment groups were assessed using a repeated measures analysis of variance. Statistical analyses were performed using SPSS version 22.0 (SPSS, Inc., Chicago, IL, USA). Probability values of $P<0.05$ were considered statistically significant.

\section{Results}

A total of 52 eyes of 52 individuals were included in this study. Depending on their preference, twenty-six cases were enrolled in the FLACS group. The remaining 26 cases were enrolled in the CPS group. No cases were excluded because of poor scan quality or complications after the surgery. Results of statistical analysis of the patient characteristics are presented on table 1.

Table 2 summarises the intraoperative outcomes of participants. The FLACS group showed a shorter surgical time than did the CPS group (FLACS: $371.31 \pm 66.26 \mathrm{~s}$ vs. CPS: $337.35 \pm 40.10 \mathrm{~s} ; \mathrm{P}=0.031$ ). In addition, the irrigation/aspiration time (I/A T) was significantly shorter when patients were treated via CPS than via FLACS (FLACS: $103.00 \pm 15.28$ s vs. CPS: $90.92 \pm 8.28 \mathrm{~s}, \mathrm{P}=0.001$ ). There was no difference in either effective phacoemulsification time (EPT) or balanced salt solution (BSS) between the groups (EPT: $P=0.536$; $B S S: P=0.093$ ). 
Figure 2 outlines BCVA and IOP outcomes. The BCVA was significantly better in each group at all timepoints after surgery (FLACS: $P<0.001$; CPS: $P<0.001)$. The IOP were similar in each group before and after surgery (FLACS: $P=0.307$; CPS: $P=0.234$ ). There were no differences in BCVA and IOP at each time point between the FLACS and CPS groups (all P >0.05).

\section{RPC density}

In the FLACS group, both whole-image RPC density and peripapillary RPC density decreased significant at all time-points after surgery (whole-image RPC density: $\mathrm{P}<0.001$; peripapillary RPC density: $\mathrm{P}<0.001$ ). Moreover, multiple comparisons revealed significant differences in the superior-hemi and inferior-hemi regions of peripapillary RPC density (superior-hemi region: $P \leq 0.001$; inferior-hemi region: $P<0.001$ ) (table 3).

In the CPS group, there were no significant decrease in both whole-image and peripapillary RPC densities at any time-point after surgery (whole-image RPC density: $P$ > 0.05; peripapillary RPC density: $P>0.05$ ). Additional multiple comparisons revealed that there were also no significant differences in peripapillary RPC density between the superior-hemi and inferior-hemi regions (superior-hemi RPC density: $\mathrm{P}>0.05$; inferior-hemi RPC density: P > 0.05) (table 3).

By comparing the FLACS and CPS group at each time-point, we found no significant differences in any of the metrics prior to surgery. However, the whole-image and peripapillary RPC densities were significantly different at day 1 (whole-image RPC density: $P=0.020$; peripapillary RPC density: $P=0.029$ ) and month 1 after the surgery (whole image RPC density: $P=0.009$; peripapillary RPC density: $P=0.005$ ). At the 3months follow-up, we did not observe significant differences (whole image RPC density: $P=0.092$; peripapillary RPC density: $P=0.070$ ). Further multiple comparisons revealed that density in the inferiorhemi region was significantly smaller in the FLACS group than in the CPS group at all time-points after the surgery (inferior-hemi RPC density: all $P<0.05$ ). However, density in the superior-hemi region was not significantly different in the FLACS group than in the CPS at all time-points after surgery (superior-hemi RPC density: all P > 0.05). (Figure 3).

\section{Discussion}

This study demonstrated that RPC in the selected regions were significantly decreased on day 1 and month 1 and 3 after surgery in the FLACS group. However, there were no significant RPC density changes in these regions in the CPS group after the surgery. Moreover, direct comparison of the FLACS and CPS groups showed that there were statistically significant differences in whole-image RPC density and peripapillary RPC density at all the time-points after the surgery. However, the differences in the wholeimage and peripapillary RPC densities were no-longer significant at the 3-month follow-up. For both the treatment groups, visual acuity improved significantly after surgery; no differences were observed at any time-point before or after the surgery between the two groups. These results suggest that the femtosecond laser pre-treatment prior to the cataract surgery was the primary reason for the observed reduction in RPC density. 
Our results are supported by previous work, which has demonstrated that a femtosecond pre-treatment during cataract surgery can cause a transient increase in IOP. Talamo et al. revealed that IOP increased linearly with an increase in the suction vacuum level and reached the maximal level at a suction pressure of $32 \mathrm{mmHg}$ using

the curved contact lens interface in porcine and cadaver eyes [24]. Zhi et al. examined retinal capillary filling in rats with an acutely elevated IOP [25]. They demonstrated that retinal blood flow diminished linearly with an increase in IOP from 30-100 mm Hg. Ecsedy et al. further determined that the application of the suction ring increased patient's IOP by up to $40 \mathrm{mmHg}$ [26]. While the IOP did decrease after removing the suction ring, it remained above baseline. 9 An elevated IOP can result in a decrease in the ocular perfusion pressure and in turn, reduce ocular blood flow [27]. Therefore, we speculate that the intense intraoperative IOP fluctuations may produce an acute ischemic-reperfusion injury to the retina, leading to retinal ganglion cell loss, axonal degeneration, and even occlusion of retinal capillaries, especially in elderly patients cataract.

With aging, the autoregulatory capacity of retinal blood vessels gradually diminishes in elderly cataract patients. However, the rate of this decline displays a wide inter-individual variability [16, 28$]$. Autoregulation serves to maintain a relatively constant blood flow on account of perfusion pressure fluctuations. Previous studies have documented that changes in retinal vascular endothelial cells, smooth muscle cells, and pericytes are a part of the aging process [29]. These changes are expected to reduce the capacity of blood vessels to vasoconstrict and vasodilate, which are essential functions to be performed for autoregulation. In accordance with these conjectures, the flow density of the optic disc has been observed to decrease with age in humans [30]. Kida et al. revealed that the nocturnal optic disc blood flow of 80-year-old participants was less than that of 20-25-year-old participants [31]. In this study, the mean age of cataract patients was $63.00 \pm 5.22$ years and $65.50 \pm 6.85$ years in the FLACS and CPS groups, respectively. The age-associated declines in the autoregulatory ability of the retinal blood vessels may decrease excessively because of femtosecond laser pre-treatment.

We further noticed that the FLACS required a longer surgical time than did the CPS group. Moreover, I/A time was significantly longer in the FLACS group. We also noticed that the BSS was greater in the FLACS group; however, this difference did not reach the threshold of statistical significance. Research has suggested that FLACS can effectively reduce EPT and CDE, properties related to the laser-assisted fragmentation and softening of the lens [32]. In our investigation, we specifically excluded patients with moderate to severe cataract and scans signal strength index of

$<50$ to acquire high-quality image; this may be why we did not observe differences in CDE and EPT between the groups in our study. However, the I/A time and TOT were significantly higher in the FLACS group. Perhaps the laser was applied in a range above and below the anterior capsule in the lines and spots inherent to the femtosecond laser [33]. The vertical application of the femtosecond laser to the cortex below the anterior capsule can cause a structural change, which can in turn make cortical cleaving hydrodissection more difficult. This remaining cortical layer also has the potential to render I/A more 
difficult to perform [34]. The prolonged I/A time and TOT of femtosecond group may have contributed to the RPC density reduction after the surgery.

It has also been shown that prostaglandin levels had increased more noticeably after FLACS than after CPS $[35,36]$. The release of prostaglandins can lead to postoperative inflammation and consequently, the breakdown of the blood-retinal barrier [35, 37 ]. Thermal damage from prolonged laser treatment [ 38 ] and mechanical microtrauma from femtosecond laser pulses [39] may also contribute to the elevated inflammation observed following FLACS than after CPS. Residual particulate lens material can also stimulate inflammation and lens fragmentation following FLACS and could additionally contribute to prostaglandin-associated inflammation [40]. Changes in inflammatory factors and prostaglandin levels may result in various retinal pathologic conditions, even the irreversible occlusion of retinal capillaries.

We also noted that the RPC density of the inferior-hemi region was significantly lower at all postoperative time-points; however, there were no significant differences in the superior-hemi region density either before or after surgery. We speculate that elevated IOP related morphological alternations in the optic disc may occur at different IOP contrast levels. Further research using a larger sample size is needed to clarify the exact underlying mechanism.

Our study possesses several potential limitations. Firstly, we were unable to assess a wider age-range of patients, and therefore our speculation on blood-vessel autoregulation could not be more deeply interrogated. Secondly, we did not assess longer-term outcomes of eye-relayed morbidity; therefore, the functional importance of FLACS associated reductions in RPC density cannot be directly assessed.

Thirdly,the number of included participants represents a relatively small sample size, and therefore, our results may have been influenced by the short follow-up time. Further longitudinal studies involving larger numbers of patients are thus needed.

In conclusion, we found a significant decrease in the RPC density after FLACS; however, there was no significant difference in the visual acuity between the two groups. Further investigation is needed to understand whether the decrease of radial peripapillary capillary density, as measured by OCTA, reflects a concern regarding the safety of FLACS. This question is especially prudent for patients with, among others, glaucoma, age-related macular degeneration, and diabetic retinopathy.

\section{Declarations}

\section{Data Availability}

The data used to support the findings of this study are available from the corresponding author on request.

\section{Conflicts of Interest}

The authors declare that there is no conflict of interest regarding the publication of this article. 
Acknowledgments

This research did not receive any specific grant from funding agencies in the public, commercial, or notfor-profit sectors.

\section{References}

1. The advanced glaucoma intervention study (AGIS) 7. The relationship between control of intraocular pressure and visual field deterioration. American Journal of Ophthalmology. 2000; 130: 429-440.

2. Gaasterland D, Tanishima T, Kuwabara T. Axoplasmic flow during chronic experimental glaucoma. 1. Light and electron microscopic studies of the monkey optic nervehead during development of glaucomatous cupping. Invest Ophthalmol Vis Sci. 1978; 17: 838-846.

3. Burgoyne $\mathrm{CF}$, et al. The optic nerve head as a biomechanical structure: a new paradigm for understanding the role of IOP-related stress and strain in the pathophysiology of glaucomatous optic nerve head damage. Prog Retin Eye Res. 2005; 24: 39-73.

4. Bonomi L, et al. Vascular risk factors for primary open angle glaucoma: the Egna-Neumarkt Study. Ophthalmology. 2000; 107: 1287-1293.

5. Leske MC, et al. Predictors of long-term progression in the early manifest glaucoma trial. Ophthalmology. 2007; 114: 1965-1972.

6. Ivers $\mathrm{KM}$, et al. In vivo detection of laminar and peripapillary scleral hypercompliance in early monkey experimental glaucoma. Invest Ophthalmol Vis Sci. 2016; 57: 388-403.

7. Sharma $S$, et al. Effect of acute intraocular pressure elevation on the minimum rim width in normal, ocular hypertensive and glaucoma eyes. Br J Ophthalmol. 2017; 102: 131-135 .

8. Popovic M, et al. Efficacy and safety of femtosecond laser-assisted cataract surgery compared with manual cataract surgery: a meta-analysis of 14567 eyes. Ophthalmology. 2016; 123: 2113-2126

9. Kerr NM, et al. Intraocular pressure during femtosecond laser pretreatment of cataract. $J$ Cataract Refract Surg. 2013; 39: 339-342.

10. Bissen-Miyajima $\mathrm{H}$, et al. Experimental observation of intraocular pressure changes during microkeratome suctioning in laser in situ keratomileusis. J Cataract Refract Surg. 2005; 31: 590-594.

11. Darian-Smith E, et al. Intraocular pressure during femtosecond laser pretreatment: Comparison of glaucomatous eyes and nonglaucomatous eyes. J Cataract Refract Surg. 2015; 41: 272-277.

12. Stulting RD, et al. Complications of laser in situ keratomileusis for the correction of myopia. Ophthalmology. 1999; 106: 13-20.

13. Arevalo JF. Retinal complications after laser-assisted in situ keratomileusis (LASIK). Curr Opin Ophthalmol. 2004; 15: 184-191.

14. Wang L, Cull GA, Fortune B. Optic nerve head blood flow response to reduced ocular perfusion pressure by alteration of either the blood pressure or intraocular pressure. Curr Eye Res. 2015; 40: 359-367. 
15. Tan PE, et al. Quantitative confocal imaging of the retinal microvasculature in the human retina. Invest Ophthalmol Vis Sci. 2012; 53: 5728-5736.

16. Jiang $X$, et al. The effect of age on the response of retinal capillary filling to changes in intraocular pressure measured by optical coherence tomography angiography. Microvasc Res. 2018; 115:12-19.

17. Li M, et al. Retinal microvascular network and microcirculation assessments in high myopia. Am J Ophthalmol. 2017; 174: 56-67.

18. Spaide RF, Klancnik JM Jr, Cooney MJ. Retinal vascular layers imaged by fluorescein angiography and optical coherence tomography angiography. JAMA Ophthalmol. 2015; 133: 45-50.

19. Shahlaee A, et al. In vivo assessment of macular vascular density in healthy human eyes using optical coherence tomography angiography. Am J Ophthalmol. 2016; 165: 39-46.

20. lafe NA, et al. Retinal capillary density and foveal avascular zone area are age-dependent: quantitative analysis using optical coherence tomography angiography. Invest Ophthalmol Vis Sci 2016; 57: 5780-5787.

21. Zahid S, et al. Fractal dimensional analysis of optical coherence tomography angiography in eyes with diabetic retinopathy. Invest Ophthalmol Vis Sci. 2016; 57: 4940-4947.

22. Tan ACS, et al. An overview of the clinical applications of optical coherence tomography angiography. Eye (Lond). 2018; 32: 262-286.

23. Kraus MF, et al. Quantitative 3D-OCT motion correction with tilt and illumination correction, robust similarity measure and regularization. Biomed Opt Express. 2014; 5: 2591-2613.

24. Talamo JH, et al. Optical patient interface in femtosecond laser-assisted cataract surgery: contact corneal applanation versus liquid immersion. J Cataract Refract Surg. 2013; 39: 501-510.

25. Zhi Z, et al. Evaluation of the effect of elevated intraocular pressure and reduced ocular perfusion pressure on retinal capillary bed flling and total retinal blood flow in rats by OMAG/OCT. MicrovasC Res. 2015; 101: 86-95.

26. Ecsedy M, et al. Effect of femtosecond laser cataract surgery on the macula. J Refract Surg. 2011; 27: 717-722.

27. Berisha F, et al. Effect of trabeculectomy on ocular blood flow. Br J Ophthalmol. 2005; 89: 185-188.

28. Wei $Y$, et al. Age-Related Alterations in the Retinal Microvasculature, Microcirculation, and Microstructure. Invest Ophthalmol Vis Sci. 2017; 58: 3804-3817.

29. Scioli MG, et al. Ageing and microvasculature. Vasc Cell. 2014; 16: 6-19.

30. Boehm AG, Koeller AU, Pillunat LE. The effect of age on optic nerve head blood Invest Ophthalmol Vis Sci. 2005; 46: 1291-1295.

31. Kida T, Liu JH, Weinreb RN. Effect of aging on nocturnal blood flow in the optic nerve head and macula in healthy human eyes. J Glaucoma. 2008; 17: 366-371.

32. Vasavada VA, et al. Comparative evaluation of femtosecond laser-assisted cataract surgery and conventional phacoemulsification in eyes with a shallow anterior chamber. $J$ Cataract Refract Surg. 2019; 45: 547-552. 
33. He L, Sheehy K, Culbertson W. Femtosecond laser-assisted cataract surgery. Curr Opin Ophthalmol. 2011; 22: 43-52.

34. Lake JC, et al. Second-wave hydrodissection for aspiration of cortical remains after femtosecond laser-assisted cataract surgery. J Cataract Refract Surg. 2018; 44: 677-679.

35. Schultz T, et al. Changes in prostaglandin levels in patients undergoing femtosecond laser-assisted cataract surgery. J Refract Surg. 2013); 29: 742-747.

36. Thomas MA, O'Grady GE, Swartz SL. Prostaglandin levels in human vitreous. Br J Ophthalmol. 1985; 69: 275-279.

37. Abell RG, Allen PL, Vote BJ. Anterior chamber flare after femtosecond laser-assisted cataract surgery. J Cataract Refract Surg. 2013; 39: 1321-1326.

38. Kessel L, et al. Post-cataract prevention of inflammation and macula edema by steroid and nonsteroidal anti-inflammatory eye drops: a systematic review. Ophthalmology. 2014; 121: 19151924.

39. Schultz T, et al. Prostaglandin release during femtosecond laser-assisted cataract surgery: main inducer. J Refract Surg. 2015; 31: 71-81.

40. Cohen SM, Davis A, Cukrowski C. Cystoid macular edema after pars plana vitrectomy for retained lens fragments. J Cataract Refract Surg. 2006; 32: 1521-1526.

\section{Tables}

Table 1: Patient demographic and clinical characteristics.

\begin{tabular}{llll}
\hline Parameters & FLACS group $(\mathrm{n}=26)$ & $\begin{array}{l}\text { CPS } \\
\text { group }(\mathrm{n}=26)\end{array}$ & P-value \\
\hline Sex, male/female (n) & $11 / 15$ & $14 / 12$ & 0.405 \\
Age (y) & $63.00 \pm 5.22$ & $65.50 \pm 6.85$ & 0.145 \\
SE (D) & $-0.136 \pm 0.56$ & $-0.217 \pm 0.68$ & 0.637 \\
CCT $(\mu \mathrm{m})$ & $548.92 \pm 25.28$ & $537.38 \pm 20.63$ & 0.077 \\
ACD $(\mathrm{mm})$ & $3.73 \pm 0.15$ & $3.70 \pm 0.14$ & 0.49 \\
AL $(\mathrm{mm})$ & $23.46 \pm 0.78$ & $23.43 \pm 0.66$ & 0.875 \\
SBP $(\mathrm{mmHg})$ & $117.65 \pm 6.69$ & $118.23 \pm 8.32$ & 0.784 \\
DBP $(\mathrm{mmHg})$ & $73.46 \pm 7.98$ & $74.19 \pm 7.96$ & 0.742 \\
MAP (mmHg) & $88.06 \pm 5.84$ & $88.87 \pm 6.58$ & 0.642 \\
MOPP (mmHg) & $48.41 \pm 4.33$ & $49.25 \pm 5.39$ & 0.535 \\
Pulse (bpm) & $76.23 \pm 6.73$ & $76.65 \pm 8.61$ & 0.844 \\
Glucose (mmol/L) & $5.32 \pm 0.46$ & $5.35 \pm 0.48$ & 0.795 \\
HbA1c (\%) & $5.06 \pm 0.53$ & $5.09 \pm 0.59$ & 0.882 \\
Cholesterol (mmol/L) & $4.43 \pm 1.05$ & $4.48 \pm 0.89$ & 0.853 \\
Triglycerides(mmol/L) & $1.32 \pm 0.23$ & $1.47 \pm 0.59$ & 0.238 \\
\hline
\end{tabular}

Means $\pm \mathrm{SD}$;

ACD: anterior chamber depth, AL: axial length, bpm: beat per minute, CCT: central corneal thickness, CPS: conventional phacoemulsification surgery, DBP: diastolic blood pressure, FLACS: femtosecond laser-assisted cataract surgery, IOP: intraocular pressure, MAP: mean arterial pressure, MOPP: mean ocular perfusion pressure, SBP: systolic blood pressure, $\mathrm{SE}$ : spherical equivalent, $\mathrm{MAP}=\mathrm{DBP}+1 / 3$ (SBP - DBP), $\mathrm{MOPP}=2 / 3 \mathrm{MAP}$ - IOP; 
Table 2: Intraoperative outcomes for all eyes in the FLACS or CPS groups.

\begin{tabular}{llll}
\hline Parameters & FLACS group & CPS group & P-value \\
\hline FLAPT (S) & $137.69 \pm 20.45$ & - & - \\
EPT (S) & $69.27 \pm 16.59$ & $64.92 \pm 14.28$ & 0.536 \\
I/A T (S) & $103.00 \pm 15.28$ & $90.92 \pm 8.28$ & 0.001 \\
BSS (ml) & $78.08 \pm 14.70$ & $71.92 \pm 10.96$ & 0.093 \\
CDE & $2.17 \pm 1.33$ & $2.35 \pm 0.67$ & 0.537 \\
TOT (S) & $371.31 \pm 66.26$ & $337.35 \pm 40.10$ & 0.031 \\
\hline
\end{tabular}

Means \pm SD;

BSS: balanced salt solution, CDE: cumulative dissipated energy, CPS: conventional phacoemulsification surgery, EPT: effective phacoemulsification time, FLACS: femtosecond laser-assisted cataract surgery, FLAPT: femtosecond laserassisted pretreatment time, I/A T: Irrigation/aspiration time, TOT: total operating time;

Table 3: Measured RPC densities for the FLACS and CPS groups.

\begin{tabular}{|c|c|c|c|c|c|c|}
\hline \multicolumn{2}{|c|}{ RPC Density (\%) } & Before surgery & \multirow{2}{*}{$\frac{1 \text { day }}{47.17 \pm 2.32}$} & \multirow{2}{*}{$\begin{array}{l}1 \mathrm{~m} \\
47.21 \pm 2.59\end{array}$} & \multirow{2}{*}{$\begin{array}{l}3 \mathrm{~m} \\
47.66 \pm 2.30\end{array}$} & \multirow{2}{*}{$\frac{P \text {-value }}{<0.001}$} \\
\hline FLACS group & Whole Image & $49.18 \pm 2.97$ & & & & \\
\hline & Peripapillary & $53.40 \pm 3.33$ & $51.37 \pm 2.65$ & $51.04 \pm 2.79$ & $51.62 \pm 2.88$ & $<0.001$ \\
\hline & Superior-Hemi & $53.96 \pm 3.80$ & $51.87 \pm 3.23$ & $51.60 \pm 2.82$ & $52.01 \pm 3.23$ & 0.001 \\
\hline & Inferior-Hemi & $52.84 \pm 3.70$ & $50.88 \pm 2.66$ & $50.48 \pm 3.15$ & $51.22 \pm 3.14$ & $<0.001$ \\
\hline \multirow{4}{*}{$\begin{array}{l}\text { CPS } \\
\text { group }\end{array}$} & Whole Image & $48.40 \pm 2.26$ & $48.99 \pm 2.66$ & $49.03 \pm 1.98$ & $49.06 \pm 3.37$ & 0.445 \\
\hline & Peripapillary & $52.89 \pm 2.89$ & $53.25 \pm 2.76$ & $53.32 \pm 2.19$ & $53.23 \pm 3.43$ & 0.812 \\
\hline & Superior-Hemi & $53.35 \pm 3.48$ & $53.48 \pm 3.10$ & $53.27 \pm 2.75$ & $53.29 \pm 3.94$ & 0.978 \\
\hline & Inferior-Hemi & $52.44 \pm 2.84$ & $53.02 \pm 2.71$ & $53.37 \pm 2.07$ & $53.17 \pm 3.29$ & 0.320 \\
\hline
\end{tabular}

Means \pm SD;

RPC: radial peripapillary capillary;

\section{Figures}

\section{Figure 1}


A $4.5 \times 4.5 \mathrm{~mm}$ image of optic disc angiogram. a: inside disc region; b: peripapillary radial peripapillary capillary region
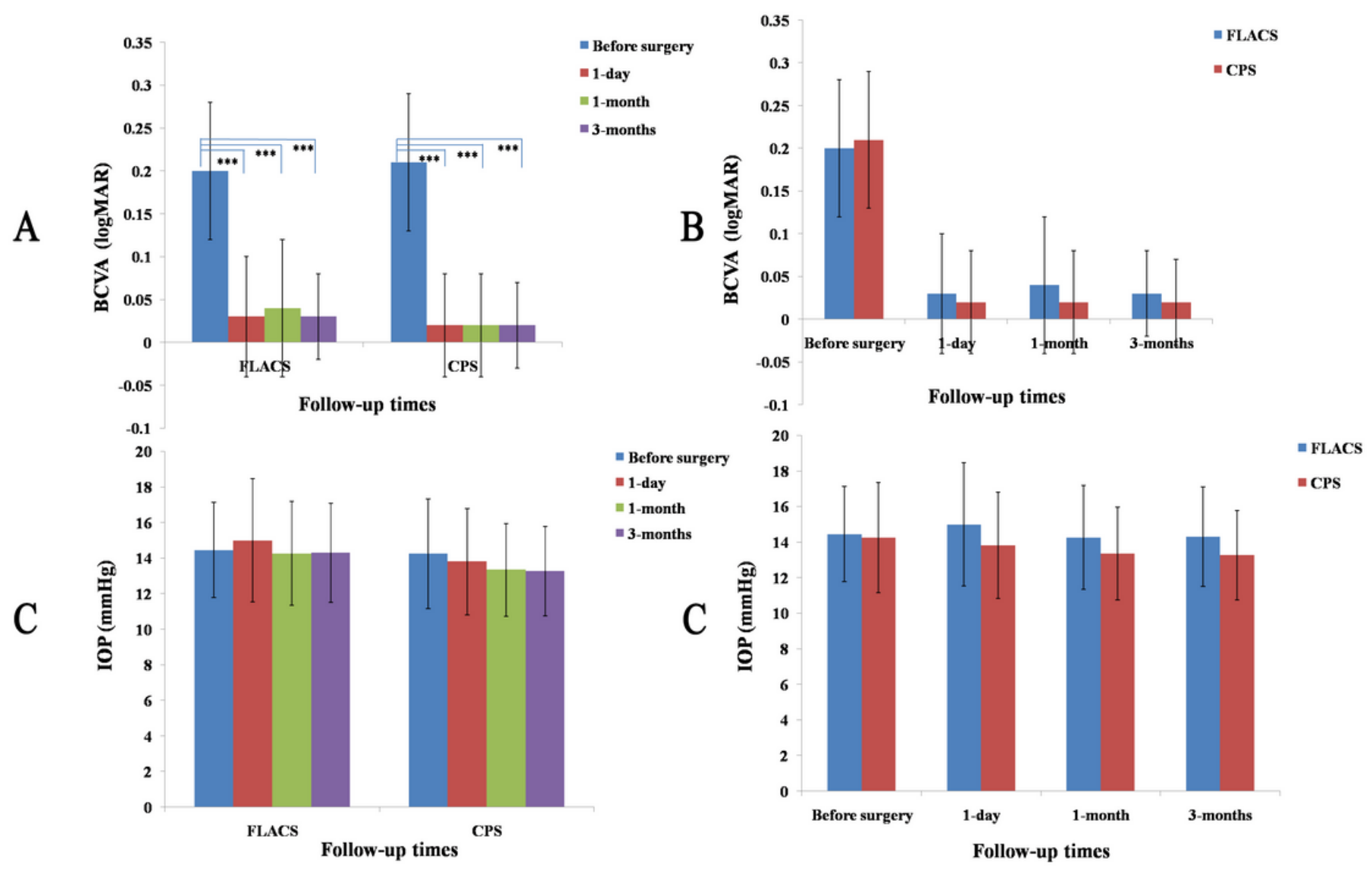

Figure 2

Pre- and postoperative changes in LogMAR BCVA and IOP of the two groups. A: Comparison of BCVA within group. B: Comparison of BCVA between groups. C: Comparison of IOP within group. D: Comparison of IOP between groups. BCVA: best-corrected visual acuity, LogMAR: logarithm of the minimum angle of resolution, IOP: intraocular pressure; ${ }^{\star \star *} \mathrm{P}<0.001$. 


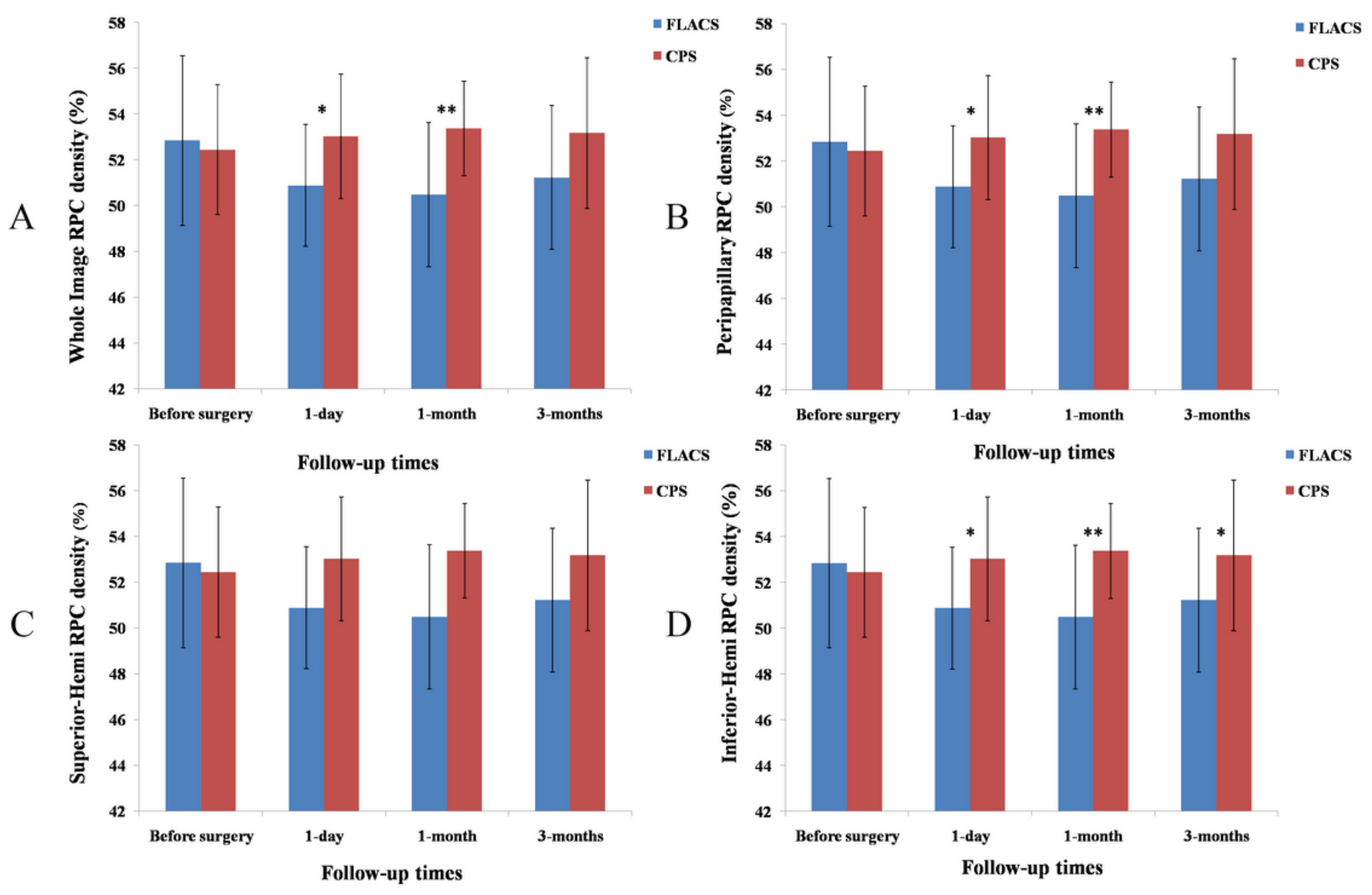

Figure 3

Pre- and postoperative changes in RPC density between groups. A: Comparison of whole-image RPC density. B: Comparison of peripapillary RPC density. C: Comparison of peripapillary RPC density of superior-hemi region. D: Comparison of peripapillary RPC density of inferior-hemi region. RPC: radial peripapillary capillary; ${ }^{*} 0.01<\mathrm{P} \leq 0.05 ; * \star \mathrm{P} \leq 0.01$ 